\title{
The Effect of Active Knee Extension in Sitting on Lumbopelvic Curvature in Individuals with Clinically Tight Hamstring Muscles: A Cross-Sectional Reliability Study
}

\author{
Musashi Yasuda, Kana Nishimoto, Masataka Hori, Tatsuya Noguchi, Hiroshi Takasaki* \\ Department of Physical Therapy, Saitama Prefectural University, Koshigaya, Japan \\ Email: *takasaki-hiroshi@spu.ac.jp
}

How to cite this paper: Yasuda, M., Nishimoto, K., Hori, M., Noguchi, T. and Takasaki, H. (2017) The Effect of Active Knee Extension in Sitting on Lumbopelvic Curvature in Individuals with Clinically Tight Hamstring Muscles: A Cross-Sectional Reliability Study. Open Journal of Therapy and Rehabilitation, 5, 139-147.

https://doi.org/10.4236/ojtr.2017.54012

Received: October 20, 2017

Accepted: November 24, 2017

Published: November 27, 2017

Copyright () 2017 by authors and Scientific Research Publishing Inc. This work is licensed under the Creative Commons Attribution International License (CC BY 4.0).

http://creativecommons.org/licenses/by/4.0/

\section{(c) (i) Open Access}

\begin{abstract}
Relative flexibility between the hamstring and lumbar extensor muscles, which can be evaluated using lumbopelvic curvature during active knee extension in sitting, can sometimes be assessed in physical therapy. However, reliability for its quantitative measure has not been established yet and its establishment was the aim of the current study. Twenty-seven individuals with clinically tight hamstring muscles were recruited. On two separate sessions, the lumbopelvic curvature was evaluated in sitting when the right knee was moved from $90^{\circ}$ flexion to $10^{\circ}$ flexion on 15 occasions using a flexible ruler by two examiners on Day 1 and one on Day 2. Lines drawn tangential to the lumbopelvic curvature were traced at T12 and S2 vertebral levels and the angle between the two vertical lines was calculated. Using Day 1 data, the minimum number of repetitions and inter-examiner reliability were assessed. Inter-session reliability was also examined. As a result, there was no statistical difference $(P>0.05)$ in the mean absolute difference between the mean value of $\mathrm{N}-1$ and $\mathrm{N}$ repetitions $(6 \leq \mathrm{N} \leq 15)$ in the lumbopelvic curvature angle, indicating that five was considered the minimum number of repetitions. Intraclass correlation coefficient (ICC) $)_{(1,5)}$ for the inter-session reliability and $\operatorname{ICC}_{(2,}$ ${ }_{5}$ for the inter-examiner reliability was 0.97 and 0.93 , respectively, indicating excellent reliability. The measure for the lumbopelvic curvature during active knee extension in sitting, which was established in the current study, will be a foundation for further research regarding the relative flexibility of the lumbar and adjunct regions.
\end{abstract}

\section{Keywords}

Lumbosacral Region, Muscle Tightness, Hamstring, Physical Examination, 
Relative Flexibility, Test-Retest Reliability

\section{Introduction}

There has been increasing interest in the role that relative flexibility between the lumbar spine and its adjunct body regions plays in the development of low back pain (LBP) [1] [2] [3]. In particular, when the hamstring muscles are relatively tighter than the lumbar extensor muscles, flexion loading to the lumbar spine is increased during movements involving hip flexion and knee extension. Thus, examination of relative flexibility of the hamstring and lumbar extensor muscles is considered an important element in the evaluation of LBP and its subgroups [4]. One clinical method used to determine lumbar spine relative flexibility is to measure the magnitude of lumbopelvic curvature during active knee extension in sitting [4]. However, a quantitative measure of lumbopelvic curvature during active knee extension in sitting has not been established yet, nor has reliability for this test been reported. Identifying subgroups who respond to specific interventions is suggested to be a priority for research in musculoskeletal physical therapy [5]. Therefore, it is necessary to establish reliability of quantitative measures for physical assessments that are used to identify specific subgroups and thus the reliability of the test to assess lumbopelvic curvature during active knee extension in sitting.

When a mean value is used as a representative value and when there is no substantial systematic bias, the mean value becomes closer to a truly representative value. However, to minimize effort of patients and examiners in the clinical practice, it is important to understand minimum number of repetitions to obtain a representative value. The number of repetitions affects results of inter-examiner reliability and inter-session reliability and therefore should be determined first. There are two possible methods to investigate the minimum number of repetitions. One is a mathematical estimation using the Spearman-Brown formula and intraclass correlation coefficients (ICC) between two repetitions. However, the mathematical estimation is not always accurate [6]. The other is to investigate differences of mean values with successive inclusion of additional repetitions [7] [8]. The latter method requires more information than the former method and thus considered more robust.

The aim of the current study was to establish reliability for measurement of lumbopelvic curvature during active knee extension in sitting by considering the minimum number of repetitions, inter-session reliability and inter-examiner reliability.

\section{Methods}

\subsection{Design}

A cross-sectional study design was used in this study. Ethical approval for the 
study was obtained from the Research Ethics Committee of the Saitama Prefectural University. All participants provided written consent before their data collection.

\subsection{Participants}

Convenience sampling of individuals with clinically tight hamstring muscles [4] [9] was undertaken via advertising in a local university between August 2015 to October 2016. Inclusion criteria were individuals: 1 ) $\geq 18$ years of age; 2 ) without lower limb symptoms or limitation of hip, knee or ankle range of motion; 3) without scoliosis; and 4) individuals who showed movement of the lumbar spine during active right knee extension with the hip in $90^{\circ}$ flexion and right ankle in full plantar flexion, indicating clinically right tight hamstring muscles [4] [9]. Individuals who had symptoms in the right lower leg during the test movement were excluded. Conditions of LBP were not included in the inclusion/exclusion criteria.

A priori sample size estimation was based on a confidence interval approach for the ICC [10] [11] [12] using the PASS 14 Power Analysis and Sample Size Software 2015 (NCSS, LLC, Kaysville, Utah, USA). Twenty-seven participants were estimated to obtain a $95 \%$ confidence interval width $=0.6-0.9($ ICC $=0.8$, either a two-way random-effect ANOVA model).

\subsection{Measurements}

\subsubsection{Primary Measure}

The primary measure was the magnitude of lumbopelvic curvature near end-range active right knee extension ( $10^{\circ}$ flexion). This was assessed using a flexible ruler and image analysis, which has been shown to have criterion-related validity when compared with radiographic analysis (ICC $=0.94$ - 0.96) [13]. Specifically, lumbopelvic curvature was traced from T12 to S2 spinous process using a flexible ruler (Shinwa Rules Co., Ltd., Tsubame, Niigata, Japan). ImageJ 1.6 software (National Institute of Mental Health, Bethesda, MD, USA) was used to draw a tangential line on the traced line of the lumbopelvic curvature at T12 and S2 vertebral levels and the angle between the two tangential lines calculated (Figure 1). The angle when each participant actively maintained the right knee extension position was defined as the lumbopelvic curvature during active knee extension in sitting. A positive value indicated lumbar kyphosis and a negative value indicated lumbar lordosis. Participants were asked to maintain lumbar position as much as possible during measurement process. The end-point of $10^{\circ}$ right knee flexion was standardized using a metal orthosis (Figure 2).

A previous study [13] used Auto CAD software to draw a vertical line on the traced line of the lumbar curvature at T12 and S2 vertebral level. However, due to the lack of Auto CAD software availability, ImageJ 1.6 software was used in the current study, with the tangential line at T12 and S2 drawn manually. A pilot study using data from the first 10 participants was undertaken to determine the length of the tangential line manually drawn at T12 and S2 level. Mean values of 
the lumbopelvic curvature were calculated for 15 repetitions of the measurement using three length conditions of the traced line at the Th12 and S2 level, $0.5 \mathrm{~mm}$, $1.0 \mathrm{~mm}$ and $1.5 \mathrm{~mm}$. Consequently, there was no significant difference $(P>$ $0.05)$ in the angle of the lumbopelvic curvature between the three conditions in a repeated-measure ANOVA. Thus, the current study used $0.5 \mathrm{~mm}$ to maximize approximation to the line drawn by Auto CAD software.
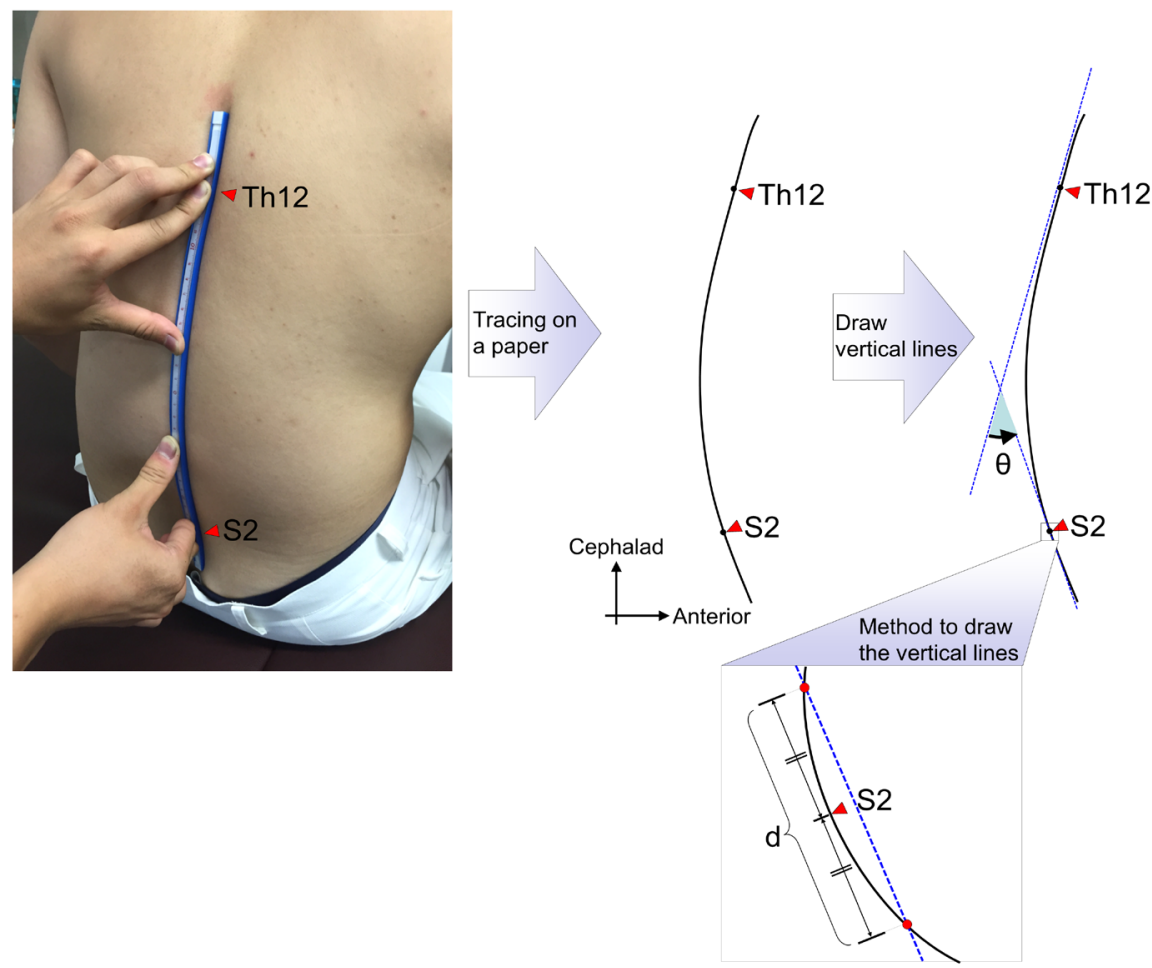

Figure 1. Schema for calculation of the angle of the lumbopelvic curvature during active knee extension in sitting $(\theta)$ on ImageJ 1.6 software.

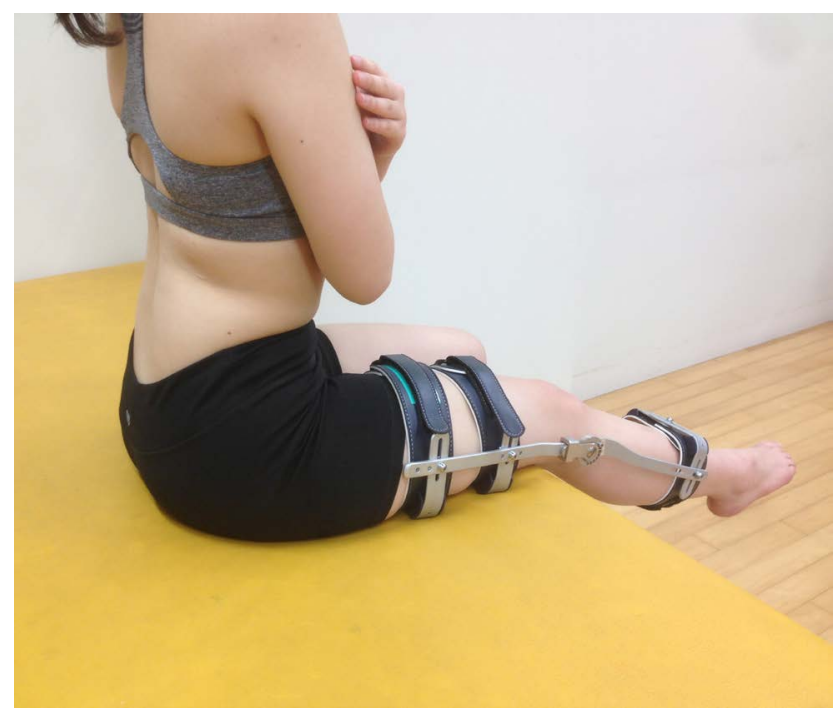

Figure 2. Standardization of knee flexion/extension angle during measurement using a metal orthosis. 


\subsubsection{Secondary Measure}

Secondary measures included gender, age, and Body Mass Index (BMI). The presence of LBP was examined considering proposed definitions of an episode of LBP and a recurrence of an episode of LBP [14] [15] [16], where participants were asked if they had pain in the low back lasting for more than 24 hours with a pain intensity of more than two on an 11-point numerical rating scale in the previous month.

\subsection{Procedures}

Data were collected on 15 repeated trials to investigate the minimum number of repetitions. Two novice examiners (students) (MY and $\mathrm{KN}$ ) participated in data collection to investigate inter-examiner reliability. Each examiner collected data and analyzed the angle of the lumbopelvic curvature separately with each examiner blind to each other. Data collection by each examiner occurred $15 \mathrm{mi}$ nutes apart, with participants resting in a lying position between trials. Examiner order was alternately allocated. To investigate inter-session reliability, data collection and analysis were undertaken by one examiner (MY). Participants were asked to attend two separate sessions on consecutive days at a similar time of a day ( \pm three hours).

\subsection{Statistics}

To determine the minimum number of repetitions, we first calculated mean values for the angle of the lumbopelvic curvature using an increasing number of successive repetitions from 2 to 15 , and then calculated the absolute difference in mean value with successive inclusion of additional repetitions (i.e. difference between mean value calculated from three vs. two repetitions; four vs. three repetitions, and so on). The minimum number of repetitions was defined as the number of repetitions at which the successive difference values plateaued (i.e. no further reduction in difference between repetitions could be achieved by the addition of more repetitions) [7] [8]. Inter-session reliability was assessed with an ICC of Model 1 and the inter-examiner reliability was assessed with an ICC of Model 2. The following criteria were used to interpret ICC values: poor reliability, <0.4; fair-good reliability, $0.4-0.75$; excellent reliability, $>0.75$ [17]. Further, the minimum detectable changes (MDC) were calculated using a standard formula:

$$
\begin{aligned}
M D C & =S E M \times 1.96 \times \sqrt{2} \\
S E M & =S D \sqrt{1-I C C}
\end{aligned}
$$

All statistical evaluation was undertaken with SPSS (IBM Corporation, Armonk, NY, USA). Statistical significance was set at $P<0.05$.

\section{Results}

There were 14 men and 13 women in the participants. Mean (SD) years of age and BMI was $21.1(2.8)$ and $21.2(3.0)$, respectively. Three participants (11.1\%) 
had LBP.

Figure 3 demonstrates the individual and group mean absolute differences between the mean value of $\mathrm{N}-1$ and $\mathrm{N}$ repetitions $(2 \leq \mathrm{N} \leq 15)$ for the lumbopelvic curvature. There was no statistical difference $(P>0.05)$ between the mean value of $\mathrm{N}-1$ and $\mathrm{N}$ repetitions $(6 \leq \mathrm{N} \leq 15)$ and therefore five was considered the minimum number of repetitions.

A post-hoc analysis was undertaken to confirm that there was no apparent systematic bias. In this study, mean values of the lumbopelvic curvature with the first five repetitions of measures (mean $[\mathrm{SD}]=9.9^{\circ}\left[14.1^{\circ}\right]$ ) and the last five repetitions of measures (mean $[\mathrm{SD}]=10.5^{\circ}\left[13.2^{\circ}\right]$ ) in data of a session for the inter-session reliability were compared with a paired sample t-test. Consequently, there was no statistical difference, $\mathrm{t}(26)=-0.65, P=0.52$ (effect size of Hedge's $\mathrm{g}$ [95\% confidence interval] $=0.04[-0.49$ to 0.57$]$ ), indicating no apparent systematic bias.

The ICC $(1,5)$ and $\operatorname{ICC}(2,5)$ (95\% confidence intervals) was 0.97 (0.92 - 0.98) and 0.93 (0.84-0.97), respectively. The ICC values indicated excellent inter-session reliability and inter-examiner reliability. The MDC was $7.3^{\circ}$.

\section{Discussion}

This study investigated various aspects of reliability for the quantitative measure of the lumbopelvic curvature during knee extension in sitting, including assessments of the minimum number of repetitions, inter-session reliability, inter-examiner reliability and MDC. The mean value of five repetitions of the

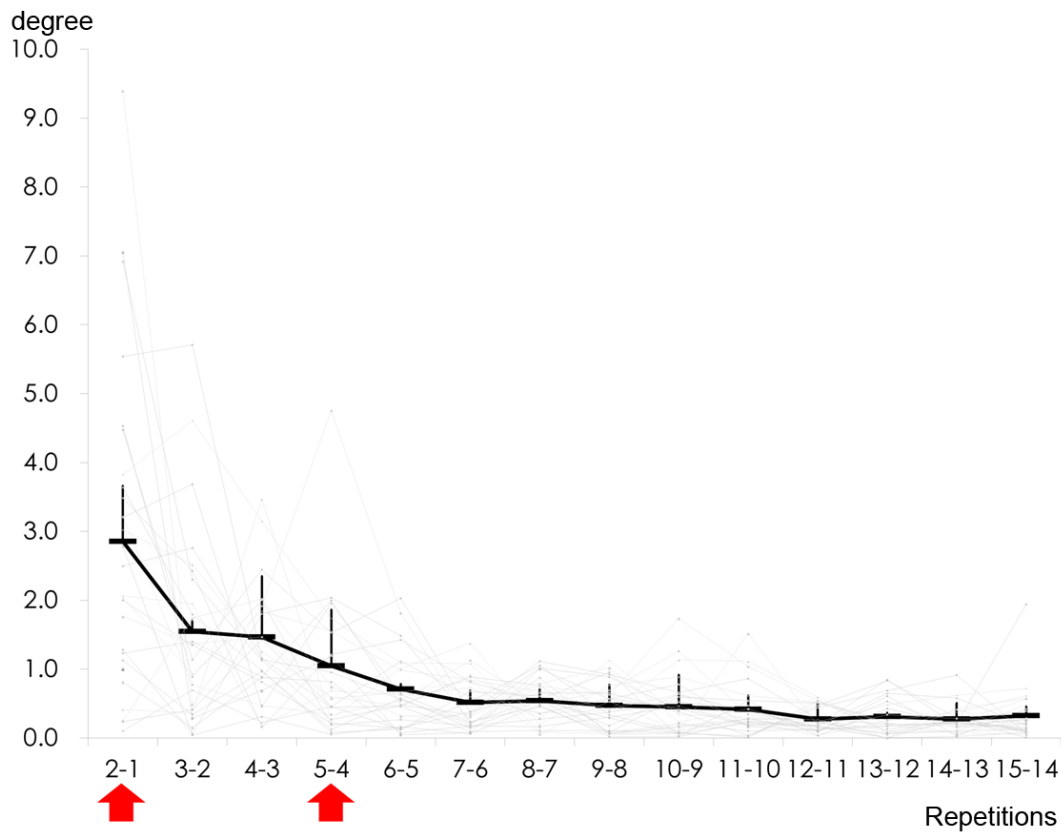

Figure 3. Group mean (black) and individual (grey) data of the absolute difference between the mean value of $\mathrm{N}-1$ and $\mathrm{N}$ repetitions $(2 \leq \mathrm{N} \leq 15)$ in the lumbopelvic curvature during active knee extension in sitting. Red arrows indicate that there were statistically significant difference $(P<0.05)$ between the mean value of $\mathrm{N}-1$ and $\mathrm{N}$ repetitions $(\mathrm{N}=2$ and 5$)$. 
measurements provides the magnitude of the lumbopelvic curvature reliably. Further, the MDC can be used to determine the treatment effect in clinical practice.

For an evaluation of stretching effect by repeating the measures, mean values of the lumbopelvic curvature with the first five repetitions of measures and the last five repetitions of measures were compared as a post-hoc analysis. Consequently, there was no statistically significant difference and the Hedge's g was 0.04 , indicating effect size of small. Therefore, it is considered that there was negligible stretching effect by repeating the measures in the current study.

Studies have demonstrated that measures of hamstring tightness are associated with LBP [18] [19] [20]. The Active Knee Extension test is considered as the gold standard assessment of the hamstring tightness [21]. However, the knee flexion/extension angle can be altered by minimum lumbopelvic movements and it is difficult to assess genuine hamstring tightness/flexibility using the Active Knee Extension test. Hamstring tightness is one of the contributing factors to lumbopelvic curvature during knee extension in sitting. A feature of the method established in the current study is to potentially reflect the flexibility balance between the lumbar spine, hip and knee regions. As relative flexibility between the lumbar spine and its adjunct regions is an important criterion for subgrouping individuals with LBP in one [4] of physiotherapy movement based classification approaches [22], this study provides preliminary information towards understanding the relationship between relative flexibility of lumbar extensor muscles to the hamstrings muscles with respect to LBP.

One example of research agenda in relation to LBP is to investigate validity. Kim et al. [2] demonstrated usefulness of assessing lumbopelvic rhythm with flexion in standing for identifying subgroups of individuals with LBP. Future studies are required to understand whether testing for the lumbopelvic curvature during active knee extension in sitting or the lumbopelvic rhythm during flexion in standing is more relevant for LBP classification. Furthermore, reliable and valid measures without specific equipment are ideal to enable clinicians to classify their patients. Therefore, future studies will be required: 1) to understand normative range of the lumbopelvic curvature during active knee extension in sitting; 2) to investigate if it is possible to identify impairment of this measure by observation or palpation of the lumbar spine movements; and 3) to understand if it is possible to estimate range of the lumbopelvic curvature during active knee extension in sitting with the metal orthosis from the test without the metal orthosis.

A limitation of the current study is the narrowly defined study population. The current study included individuals who were clinically assumed to have tight hamstring muscles but they did not have lower limb symptoms or limitation of hip, knee or ankle range of motion. Conditions of LBP were not included in the inclusion/exclusion criteria. Therefore, it can be possible to apply the measurement method established in the current study to individuals with and without LBP when individuals are assumed to have tight hamstring muscles. 
However, reliability of the measurement method in the current study was not established in individuals who had referred pain into the limb. Thus, careful selection of individuals would be required to use the measurement method established in the current study for future research.

\section{Conclusion}

The current study established inter-session and inter-examiner reliability for the assessment of lumbopelvic curvature during knee extension in sitting, as a surrogate measure of relative flexibility of the hamstrings compared to lumbar extensors muscles.

\section{Acknowledgements}

The authors acknowledge Dr. Toby Hall for help with peer review prior to submission of this paper.

\section{References}

[1] Gombatto, S.P., Collins, D.R., Sahrmann, S.A., Engsberg, J.R. and Van Dillen, L.R. (2007) Patterns of Lumbar Region Movement during Trunk Lateral Bending in 2 Subgroups of People with Low Back Pain. Physical Therapy, 87, 441-454. https://doi.org/10.2522/ptj.20050370

[2] Kim, M.-h., Yoo, W.-G. and Choi, B.-R. (2013) Differences between Two Subgroups of Low Back Pain Patients in Lumbopelvic Rotation and Symmetry in the Erector Spinae and Hamstring Muscles during Trunk Flexion When Standing. Journal of Electromyography \& Kinesiology, 23, 387-393.

https://doi.org/10.1016/j.jelekin.2012.11.010

[3] Harris-Hayes, M. and Van Dillen, L.R. (2009) The Inter-Tester Reliability of Physical Therapists Classifying Low Back Pain Problems Based on the Movement System Impairment Classification System. $P M \& R, 1,117-126$.

https://doi.org/10.1016/j.pmrj.2008.08.001

[4] Sahrmann, S.A. (2001) Diagnosis and Treatment of Movement Impairment Syndromes. Mosby, St Louis.

[5] Foster, N.E., Dziedzic, K.S., Van Der Windt, D.A.W.D., Fritz, J.M. and Hay, E.M. (2009) Research Priorities for Non-Pharmacological Therapies for Common Musculoskeletal Problems: Nationally and Internationally Agreed Recommendations. BMC Musculoskeletal Disorders, 10, 3. https://doi.org/10.1186/1471-2474-10-3

[6] Allen, D.D., Ni, P. and Haley, S.M. (2008) Efficiency and Sensitivity of Multidimensional Computerized Adaptive Testing of Pediatric Physical Functioning. Disability and Rehabilitation, 30, 479-484. https://doi.org/10.1080/09638280701625484

[7] Swait, G., Rushton, A.B., Miall, R.C. and Newell, D. (2007) Evaluation of Cervical Proprioceptive Function: Optimizing Protocols and Comparison between Tests in Normal Subjects. Spine (Phila Pa 1976), 32, E692-E701. https://doi.org/10.1097/BRS.0b013e31815a5a1b

[8] Takasaki, H., Treleaven, J., Johnston, V. and Jull, G. (2012) Minimum Repetitions for Stable Measures of Visual Dependency Using the Dot Version of the Computer-Based Rod-Frame Test. Manual Therapy, 17, 466-469.

https://doi.org/10.1016/j.math.2012.02.013 
[9] Kendall, F.P., McCreary, E.K., Provance, P.G., Rodgers, M.M. and Romani, W.A. (2005) Muscles, Testing and Function with Posture and Pain. Lippincott Wiliams \& Wilkins, Baltimore.

[10] Shrout, P.E. and Fleiss, J.L. (1979) Intraclass Correlations: Uses in Assessing Rater Reliability. Psychological Bulletin, 86, 420-428. https://doi.org/10.1037/0033-2909.86.2.420

[11] Bartko, J.J. (1966) The Intraclass Correlation Coefficient as a Measure of Reliability. Psychological Reports, 19, 3-11. https://doi.org/10.2466/pr0.1966.19.1.3

[12] Bonett, D.G. (2002) Sample Size Requirements for Estimating Intraclass Correlations with Desired Precision. Statistics in Medicine, 21, 1331-1335. https://doi.org/10.1002/sim.1108

[13] Eslam, B., Aslan, K., Zahra, M. and Saleh, B. (2012) An Innovative Software Method for Measuring Lumbar Lordosis. Annals of Biological Research, 3, 204-213.

[14] de Vet, H.C., Heymans, M.W., Dunn, K.M., et al. (2002) Episodes of Low Back Pain: A Proposal for Uniform Definitions to Be Used in Research. Spine (Phila Pa 1976), 27, 2409-2416. https://doi.org/10.1097/00007632-200211010-00016

[15] Stanton, T.R., Latimer, J., Maher, C.G. and Hancock, M.J. (2011) A Modified Delphi Approach to Standardize Low Back Pain Recurrence Terminology. European Spine Journal, 20, 744-752. https://doi.org/10.1007/s00586-010-1671-8

[16] Stanton, T.R., Latimer, J., Maher, C.G. and Hancock, M.J. (2009) Definitions of Recurrence of an Episode of Low Back Pain: A Systematic Review. Spine (Phila Pa 1976), 34, E316-E322. https://doi.org/10.1097/BRS.0b013e318198d073

[17] Fleiss, J.L. (1986) The Design and Analysis of Clinical Experiments. John Wiley and Sons, New York.

[18] Kellis, E., Ellinoudis, A. and Kofotolis, N. (2015) Hamstring Elongation Quantified Using Ultrasonography during the Straight Leg Raise Test in Individuals with Low Back Pain. PM\&R, 7, 576-583. https://doi.org/10.1016/j.pmrj.2014.12.011

[19] Stutchfield, B.M. and Coleman, S. (2006) The Relationships between Hamstring Flexibility, Lumbar Flexion, and Low Back Pain in Rowers. European Journal of Sport Science, 6, 255-260. https://doi.org/10.1080/17461390601012678

[20] Nourbakhsh, M.R., Arabloo, A.M. and Salavati, M. (2006) The Relationship between Pelvic Cross Syndrome and Chronic Low Back Pain. Journal of Back and Musculoskeletal Rehabilitation, 19, 119-128. https://doi.org/10.3233/BMR-2006-19403

[21] Davis, D.S., Quinn, R.O., Whiteman, C.T., Williams, J.D. and Young, C.R. (2008) Concurrent Validity of Four Clinical Tests Used to Measure Hamstring Flexibility. The Journal of Strength \& Conditioning Research, 22, 583-588. https://doi.org/10.1519/JSC.0b013e31816359f2

[22] Karayannis, N., Jull, G. and Hodges, P. (2012) Physiotherapy Movement Based Classification Approaches to Low Back Pain: Comparison of Subgroups through Review and Developer/Expert Survey. BMC Musculoskeletal Disorders, 13, 24. https://doi.org/10.1186/1471-2474-13-24 